\title{
NORMS AND DETERMINANTS OF PRODUCTS OF \\ LOGARITHMIC FUNCTIONS OF POSITIVE SEMI-DEFINITE OPERATORS
}

\author{
MITSURU UCHIYAMA
}

Abstract. Let $A, B$ be bounded positive semi-definite operators (matrices) on a Hilbert space. We will show

$$
\|\log (1+A) \log (1+B)\| \leqslant\left\{\log \left(1+\|A B\|^{\frac{1}{2}}\right)\right\}^{2},
$$

and

$$
\|\log (1+B) \log (1+A) \log (1+B)\| \leqslant\left\{\log \left(1+\|B A B\|^{\frac{1}{3}}\right)\right\}^{3} .
$$

Further we will prove the corresponding determinantal inequalities.

Mathematics subject classification (1991): Primary 47A30; Secondry 15A15, 15A45.

Key words and phrases: Norm, determinant, positive semi-definite operator, logarithmic function, operator monotone function, majorization.

\section{REFERENCES}

[1] T. ANDO, Majorizations, and inequalities in matrix theory,, Linear Algebra Appl. 199 (1994), 17-67.

[2] R. BHATIA, Matrix Analysis, Springer-Verlag, New York, 1996.

[3] T. FuRUTA,, Norm inequalities equivalent to Löwner-Heinz theorem, Rev. Math. Phys. 1 (1989), $135-137$.

[4] F. HIAI, Log-majorizations and norm inequalities for exponential operators, in Linear Operators, Banach center Publications 38 (1997), 119-181. 